\title{
A Full-chain OpenSim Model and Its Application on Posture Analysis of an Overhead Drilling Task
}

\author{
Jing Chang ${ }^{1}$, Damien Chablat ${ }^{2}$, Fouad Bennis ${ }^{1}$, and Liang $\mathrm{Ma}^{3}$ \\ 1 Ecole Centrale de Nantes, Laboratoire des Sciences du Numérique de Nantes \\ (LS2N), UMR CNRS 6004, 44321 Nantes, France \\ 2 CNRS, Laboratoire des Sciences du Numérique de Nantes (LS2N), UMR CNRS \\ 6004, 44321 Nantes, France damien.chablat@cnrs.fr \\ 3 Department of Industrial Engineering, Tsinghua University, Beijing, 100084, \\ P.R.China
}

\begin{abstract}
Biomechanical motion simulation and kinectic analysis of human joints and muscles provide insights into Musculoskeletal disorders. OpenSim is an open-source platform that give easy access to biomechanical analysis, especially of muscles. The biomechanical analysis in OpenSim is based on pre-defined human models. Among the dozens of models available right now, none covers the muscles and joints of all the body parts. In view of the fact that most human motions are systemic, the lack of a comprehensive model prohibits synthesized and systematical biomechanical analysis. The aim of this research is to develop an OpenSim model which enables the full-chain dynamic analysis of tasks involving multi-bodies. The model is developed based on two existing models. It consists of 45 body segments, 424 muscles and 39 degrees of freedom. The model was then used to simulate an overhead drilling task. Six drilling postures are analyzed, and the estimated joint moments and muscle activations are compared. Key words: Biomechanical analysis; OpenSim model; joint moment; muscle activation; overhead work; drilling posture; posture analysis.
\end{abstract}

\section{Introduction}

Biomechanical analysis of human positions and motions enables investigations on the neuromusculoskeletal system even beyond experiments. It provides insights into many concerned issues, such as musculoskeletal Disorders (MSDs), which makes up the vast proposition of the occupational diseases [1. When conducting biomechanical analyses, we have to bear in mind that human body is systematic: bodies and muscles are inter-connected as a whole kinematic chain. The state of one part may influent another without physical contact. For example, in a task of self-balance, the arm motions would influence the transversal pelvisthorax moment up to $30 \%$ [2. The full kinematic chain should be considered in biomechanical analysis. 
Over the past decades, many tools have been developed for biomechanical simulation and analysis. OpenSim [3] is one of the virtual human modeling software that have been widely used [4/5. There are immense amount of data and dozens of musculoskeletal models available, such as the running model 6 which includes muscles of the lower extremities and the torso, the lumbar spinal model 7. which contains the eight main muscle groups of the lumbar spine, the arm model 8 that covers 50 muscles on the right upper extremity. However, no musculoskeletal model has included the full kinematic chain of human body. There is a need of a full-chain model that enables synthesized and systematical biomechanical analysis. For example, using a muscle fatigue model [9] and a recovery model [10, it is possible to determine the maximum working time by knowing, the maximum voluntary contraction, the fatigue and recovery parameters as well as the activation rate of each muscle. With this model, we can also do posture optimization using worker fatigue and comfort indexes [11] as well the maximum endurance time for static posture [12] or dynamic posture [13]. This method was partially applied in an OpenSim model to study fatigue while walking in 14.

The aim of this research is to develop a full-chain OpenSim musculoskeletal model that includes segments and muscles of torso and all limbs. The model is then used to simulate an overhead drilling task. Six different postures are analyzed, and comparisons are made in view of joint moments and muscle efforts. Specially attention is paid to the full chain effect.

Next section presents the full-chain OpenSim musculoskeletal model. The simulation of an overhead task is reported in Section 3. Section 4 is devoted to the analysis of the simulation results. Finally, Section 5 presents a discussion of results and their application for ergonomic analysis.

\section{Model development}

The full-chain model is developed based on two existing models: Raabe's Fullbody Lumbar-spinal model [15] and Saul's 7 degree-of -freedom arm model [16]. The former includes muscles of the lumbar and the lower extremities, with 21 segments, 30 degrees-of-freedom, and 324 muscle actuators. It is characterized by a detailed description of the trunk musculature. Its geometry is obtained from a male of $180 \mathrm{~cm}$ and $75.3 \mathrm{~kg}$. The latter, Saul's Arm model represents the right shoulder, arm and hand of a male of $177 \mathrm{~cm}$ and $75.0 \mathrm{~kg}$, with 7 segments and 50 muscles.

To build up the full-chain model, we conjoint the two models above, and reconstruct the left upper extremity to endue it with the same segment structure and musculature as the right one. The entire model consists of 424 muscles, 46 body segments, with 39 degrees of freedom, shown in Figure 2 Its segment mass parameters are derived from the 3-D scanned geometries of a 31-year-old male of $177 \mathrm{~cm}$ and $77.0 \mathrm{~kg}$ [17. Validation tests were performed to validate this model with an ART motion capture system. Only the muscles of the neck are not present in this model which does not allow to include the movements of the head to see the task to be carried out in our study. The OpenSim model is 


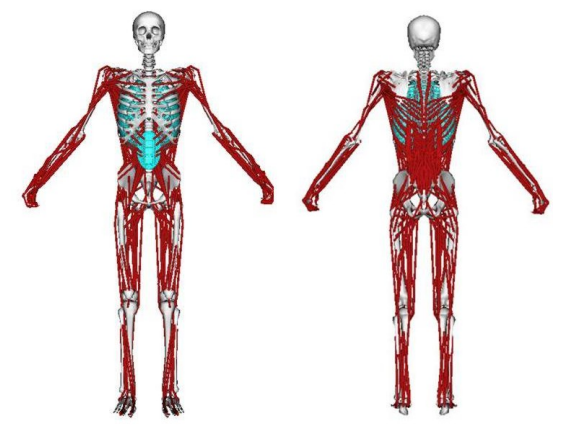

Fig. 1. The whole-body model visualized in OpenSim

defined in an XML file with links to the bone geometry for graphics rendering. The position of the motion capture markers can also be changed.

\section{Simulation of an Overhead task}

\subsection{Overhead work}

Overhead work is constantly accused by epidemiologists for its close relationship with shoulder disorders [1819. It is always accompanied by abnormal postures (for example, upper arm flexion or adduction 60 [18]) and forceful exertions. For some occupations like welder [20] and driller [21], these tasks are sometimes impractical to be avoided. An alternative plan would be to optimize the working posture.

An optimized posture is characterized by lower physical load. When estimating posture loads, attention should be payed to the integrity of human body. There is a possibility that some postures would reduce load from one part of body while bring in extra load to another.

In this study, we use the full-chain OpenSim model to simulate a typical overhead drilling task. Six task postures are analyzed. In each case, joint moments and muscle activations are checked. The sum of activations of all muscles is viewed as the index of posture load. Hypothesis is made that the index varies with different postures.

\subsection{Simulation setup}

Overhead drilling is a common task in industry. Workers hold a driller in hand while applying an force upwards. For a specific task, workers' drilling postures vary with the spatial position of the target point. Postures that vary in the sagittal plane are the main concerns [22]. In this study, we simulate an overhead drilling task about $30 \mathrm{~cm}$ higher over head. Six postures are applied, which represents 6 possible combinations of two factors: reach height (with/without 


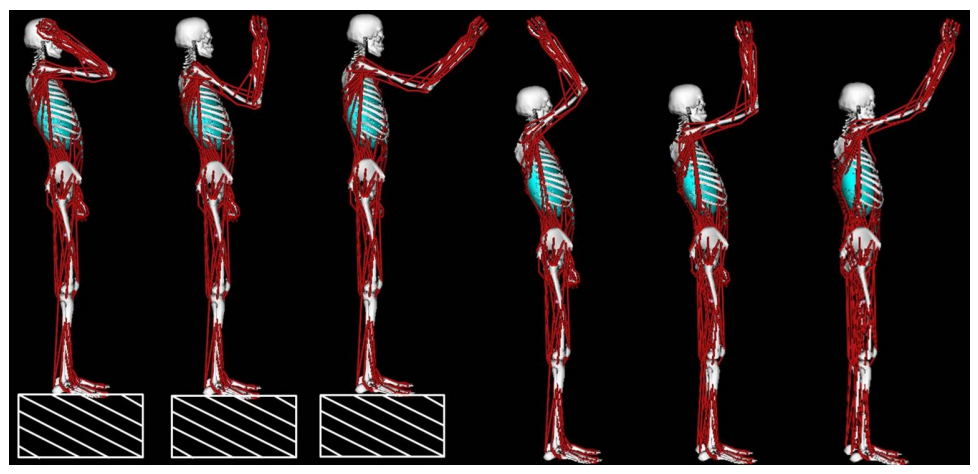

Fig. 2. Simulations of six drilling postures where L means a low hand posture and H a high hand posture with respect to the human body. From left to right: low close reach (L-C); low middle reach (L-M); low far reach (L-F); high close reach (H-C); high middle reach $(\mathrm{H}-\mathrm{M})$; high far reach $(\mathrm{H}-\mathrm{F})$.

stepladder) and reach distance (close reach/middle reach/far reach), shown in Figure 3.2

The "stepladder" is $30 \mathrm{~cm}$ high. It enables the "low-reach" postures with the wrist height at eye-level in comparison with the "high-reach" ones. In aspects of the reach distance, "close-reach", "middle-reach", "far-reach" are used to denote positions with wrist aligned with the pelvis mass center, aligned with tiptoes, and $15 \mathrm{~cm}$ further away from tiptoes, respectively. Settings of the model's joint space is presented in Table 3.2. The unmentioned joint coordinates are set to zero. An external force of $44 \mathrm{~N}$ is applied downwards on the proximal row of the

Table 1. Model joint space settings. [Unit: degree]

\begin{tabular}{l|l|llllll}
\hline \multirow{2}{*}{ Coordinates } & \multicolumn{1}{|c|}{$\begin{array}{l}\text { Permitted joint mo- } \\
\text { tions }\end{array}$} & \multicolumn{7}{|c}{ Postures } \\
\cline { 3 - 8 } & & L-C & L-M & L-F & H-C & H-M & H-F \\
\hline Flex_extension & Lumbar extension & 5 & 5 & 0 & 5 & 5 & 0 \\
Elv_angle & Shoulder adduction & 80 & 80 & 80 & 80 & 80 & 80 \\
Shoulder_elv & Shoulder elevation & 75 & 50 & 70 & 120 & 100 & 110 \\
Elbow_flexion & Elbow flexion & 145 & 120 & 60 & 80 & 70 & 40 \\
Flexion & Wrist flexion & -45 & -45 & -45 & -45 & -45 & -45 \\
Deviation & Wrist deviation & 0 & -5 & -10 & 0 & -5 & -10 \\
\hline
\end{tabular}

right hand, which simulates the load of a $2.27 \mathrm{~kg}$ portable drill together with a compression force of $22 \mathrm{~N}$ [22]. Ground reaction force is applied evenly on the two feet . 
Inverse dynamics are conducted, which computes joint moments; muscle forces and activations are estimated on optimizing the sum of total muscle activations.

\section{Results of Simulation}

Shoulder moment is the main concern of the overhead work. The simulation with the Whole-body OpenSim model permits not only the estimation of the shoulder elevation moment but a comprehensive overview of joint moments in all coordinates, as shown in Table 4 .

Table 2. Estimated joint moments of six simulations. [Unit: N.m]

\begin{tabular}{l|llllll}
\hline \multicolumn{1}{c|}{$\begin{array}{c}\text { Coordi- } \\
\text { nates }\end{array}$} & \multicolumn{6}{|c}{ Postures } \\
\cline { 2 - 6 } & L-C & L-M & L-F & H-C & H-M H-F \\
\hline $\begin{array}{l}\text { Shoulder } \\
\text { elevation }\end{array}$ & 12.27 & 13.87 & 13.90 & 11.58 & 14.39 & 13.17 \\
$\begin{array}{l}\text { Shoulder } \\
\text { rotation } \\
\text { Shoulder } \\
\text { adduction }\end{array}$ & -7.78 & -6.60 & -6.96 & -5.95 & -7.26 & -6.66 \\
$\begin{array}{l}\text { Elbow } \\
\text { flexion }\end{array}$ & -2.48 & -0.43 & 0.65 & -0.39 & -0.27 & 0.62 \\
$\begin{array}{l}\text { Hand flex- } \\
\text { ion }\end{array}$ & -3.10 & -3.92 & -2.03 & -4.00 & -4.25 & -3.24 \\
$\begin{array}{l}\text { Hand de- } \\
\text { viation }\end{array}$ & 2.07 & 9.97 & -4.41 & -13.40 & 9.69 & -4.64 \\
$\begin{array}{l}\text { Hand } \\
\text { pronation }\end{array}$ & 6.38 & -1.69 & -6.27 & 4.34 & 0.06 & -3.47 \\
\hline
\end{tabular}

Results shows that as far as the shoulder, the rotation moment is considerable beside of the elevation moment. The high close reach posture is estimated to lead to the least shoulder moment (17.92 N.m in total), while the high middle reach posture is related to the most (21.92 N.m in total). The elbow flexion moment varies substantially (from 2.40 N.m to 7.26 N.m). So is the wrist moment, which varies from $11.35 \mathrm{~N} . \mathrm{m}$ to $21.74 \mathrm{~N}$.m. It is notable that it is the high close reach posture which brings about the least shoulder moment that leads to the largest wrist moment.

Muscle activations are computed on optimizing the sum of total muscle activation while counteracting the joint moments. Table 4 presents the sum of activations of some muscle groups and of whole-body muscles in the six simulations. As a whole, the sum of all muscles' activations varies from 11.88 to 29.35, which indicates a variation of average muscle activation from $2.8 \%$ to $6.9 \%$. This 
suggests that the muscles exert efforts of different levels in different postures. Posture optimization would be a efficient way to interfere with muscle-related disorders.

The upper arm and shoulder muscle group (shown in Figure 3(a) is the primary concern of overhead works. The simulation results show that the average activation of muscles in this group varies from $2.2 \%$ (low close reach) to $8.6 \%$ (high far reach). It can also be figured out that the muscles of lower arm could be activated in varying degrees as well (averages vary from $1.2 \%$ to $8.6 \%$ ).

The simulation results also highlight the roles played by muscles of latissimus dorsi (shown in Figure 3(b) and hip abductor (shown in Figure 3(c)p. Significantly asymmetrical activation levels are shown between left and right side of the two muscle groups. For the latissimus dorsi, muscles of the right side are more activated than that of the left. And for the hip abductor, muscles of the left side are intensively activated (about $16.8 \%$ in average in every posture) while that of the right side are almost relaxed (about $0.3 \%$ to $0.4 \%$ in average). It is also notable that the activations of the right latissimus dorsi muscles vary with postures. For the low middle reach and the high middle reach, right latissimus dorsi shows far less activation than in other postures.

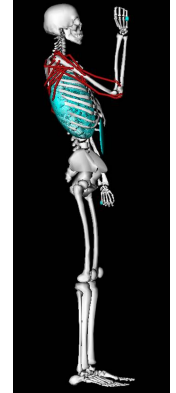

(a) Upper arm- (b) shoulder

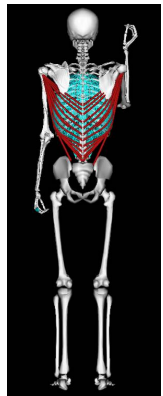

Latissimus dorsi

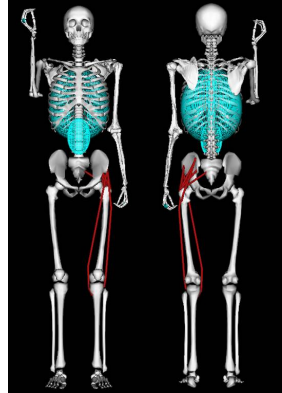

(c) Hip abductor

Fig. 3. Locations of three muscle groups

As far as overhead works, the activation of some specific muscles such as the biceps, the triceps, the deltoids have concerned researchers. In the current study, the activations of these muscles are estimated, as shown in Table 4. The biceps and posterior deltoids are not significantly activated while the anterior deltoids exerts considerable efforts in all the six simulations. The lateral deltoids is activated enormously with high close reach and high far reach posture, whereas makes trivial exertions with low middle reach posture. The long head of triceps does not exert markable effort except with the low middle reach posture; the medial and short head of triceps are most activated with high close posture (about 30\%), and least activated with low close reach posture (about $0.4 \%$ ). 
Table 3. Estimated muscle activations of muscle groups

\begin{tabular}{l|c|llllll}
\hline \multirow{2}{*}{ Muscle group } & No. of muscles & \multicolumn{5}{|c}{ Mean muscle activation } \\
\cline { 3 - 8 } & & L-C & L-M & L-F & H-C & H-M & H-F \\
\hline Right lower arm & 18 & $6.88 \%$ & $3.96 \%$ & $1.20 \%$ & $8.60 \%$ & $3.18 \%$ & $1.42 \%$ \\
Right upper arm-shoulder & 22 & $2.18 \%$ & $4.92 \%$ & $8.18 \%$ & $8.31 \%$ & $6.38 \%$ & $8.59 \%$ \\
Right Latissimus dorsi & 14 & $82.29 \%$ & $0.39 \%$ & $81.64 \%$ & $40.95 \%$ & $0.43 \%$ & $78.97 \%$ \\
Left Latissimus dorsi & 14 & $0.09 \%$ & $0.06 \%$ & $0.84 \%$ & $0.10 \%$ & $0.06 \%$ & $0.65 \%$ \\
Right hip abductor & 10 & $0.34 \%$ & $0.32 \%$ & $0.41 \%$ & $0.38 \%$ & $0.32 \%$ & $0.37 \%$ \\
Left hip abductor & 10 & $16.77 \%$ & $16.77 \%$ & $16.77 \%$ & $16.77 \%$ & $16.77 \%$ & $16.77 \%$ \\
Whole-body & 424 & $5.81 \%$ & $2.80 \%$ & $6.43 \%$ & $5.29 \%$ & $3.62 \%$ & $6.92 \%$ \\
\hline
\end{tabular}

Table 4. Estimated activations of individual muscles

\begin{tabular}{l|llllll}
\hline \multirow{2}{*}{ Muscle } & \multicolumn{5}{|c}{ Muscle activation rate } \\
\cline { 2 - 7 } & L-C & L-M & L-F & H-C & H-M & H-F \\
\hline Biceps long head & $0.00 \%$ & $0.36 \%$ & $0.00 \%$ & $0.00 \%$ & $0.36 \%$ & $0.00 \%$ \\
Biceps short head & $0.39 \%$ & $0.36 \%$ & $0.00 \%$ & $0.00 \%$ & $0.36 \%$ & $0.00 \%$ \\
Anterior deltoids & $22.06 \%$ & $18.93 \%$ & $24.37 \%$ & $13.37 \%$ & $36.98 \%$ & $19.95 \%$ \\
Lateral deltoids & $19.69 \%$ & $0.00 \%$ & $19.02 \%$ & $100.00 \%$ & $7.70 \%$ & $66.49 \%$ \\
Posterior deltoids & $0.39 \%$ & $0.36 \%$ & $0.46 \%$ & $0.43 \%$ & $0.36 \%$ & $0.42 \%$ \\
Triceps long head & $0.39 \%$ & $8.98 \%$ & $0.00 \%$ & $0.00 \%$ & $0.00 \%$ & $0.00 \%$ \\
Triceps medial head & $0.39 \%$ & $5.83 \%$ & $16.15 \%$ & $30.54 \%$ & $13.28 \%$ & $18.39 \%$ \\
Triceps short head & $0.39 \%$ & $5.72 \%$ & $15.69 \%$ & $30.00 \%$ & $13.01 \%$ & $17.06 \%$ \\
\hline
\end{tabular}




\section{Discussions}

\subsection{The full chain effect}

When evaluating work loads of the shoulder joint, ergonomists pay much attention to the moment of shoulder elevation and abduction, rather than the rotation 1819. For example, Anton et all. 22. studied an overhead drilling task with similar posture configurations with the current simulation, in which the shoulder elevation moments that are calculated from measurements taken on still video frames are used as the only indicators of posture loads, whereas results of the current simulation imply that shoulder rotation moment is also considerable. To evaluate the moments of multi-degrees-of-freedom joints, the use of refined musculoskeletal models are strongly suggested.

The full-chain effect is underlined by the simulation results. Significant activations of the right hip adductors go with all the six postures, hypothetically because of the asymmetric loads between the left and right arm. Intensive activations of the right latissimus dorsi are found in postures with forward or backward arm reach, other than the neutral. This result implies the possible relationship between back loads and arm positions.

Low back pain is an important and costly health problem in industry 2324 . Among all occupations, the highest recurrence rate is reported in nurses and drivers [25]26]. Both of the two occupations require lots of forward or backward arm reaches. Among the enormous efforts to study the ergonomic risk factors of low back pain 27/28, very little has been made on the arms. We suggest more attention to arms with regards to low back pain, and also a synthesized point of view in future ergonomic researches.

\subsection{The full-chain model as a way of ergonomic posture analysis}

One of the main findings of the overhead work simulation is that sum of all muscles' activation level varies significantly with different working postures. Here the muscle activations are estimated while solving the muscle-moment redundancy problem. Currently, the commonly used methods for this problem include static optimization (SO), computed muscle control (CMC) and neuromusculoskeletal tracking (NMT) 29. These methods are based on optimizations to minimize a given performance criterion. For $\mathrm{SO}$ and $\mathrm{CMC}$, the criterion is the sum of squares of muscle activations [30, and for NMT, the criterion is the cumulative muscle efforts expressed in a cost function (see equation 11 in Seth \& Pandy, (2007) 31]). The current study takes the SO method, which incorporates timeinstant instead of time-dependency cases. It is very computationally efficient, and as concluded by Anderson and Pandy [30, it provides reasonable predictions of muscle forces and is hereby effective. The generated muscle activation could fairly be used as a representation of muscle effort.

The posture analysis benefits also from the whole-body muscle model. As illustrated above, the full-chain effect should be considered in physical assessments. The engagement of the whole-body muscle model would avoid the case 
that the position reduces efforts on one part while bring in extra efforts on another. The sum of all muscles' activation can be an good indicator of posture load when conducting posture analysis.

\subsection{Overhead working}

Simulations of the six working postures indicate that the forward/backward reach (the L-C, L-F, H-C and H-F) postures are much more effort-demanding than the neutral reach (the L-M and H-M) postures (Table 4 ) in the overhead drilling task. This result suggests that the efforts spent on horizontal body balance takes a great portion in total efforts. The study of Maciukiewicz et al. (2016) 32 indicates that for upward overhead drilling, further forward reach distance results in greater muscle demands (measured by EMG of upper extremity muscles). Similar results were reported by Shin \& Yoo [33: overhead work with a forward reach distance of $30 \mathrm{~cm}$ leads to stronger EMG activities of upper trapezius, lower trapezius, anterior deltoid and serratus anterior than that with the distance of $15 \mathrm{~cm}$.

The EMG activity of anterior deltoid was found to be less intensive with backward reach overhead drilling posture than that with neutral reach ones by Anton (2001) 22. The finding is different from the result of current simulation (Table 4). Anton's work was based on an experimental task simulation with 20 volunteers. One explanation is that in experiments, the subjects would turn their head right and look up towards the drilling point. Whereas in our simulation, no head movement is considered due to model limitation (Figure 4). The small difference would probably influent the anterior deltoid, which works to connect the neck and shoulder. Future work should be done to refine the movement of head and neck of the full-chain model.

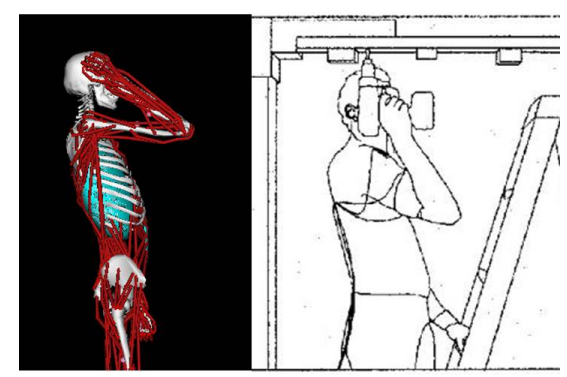

Fig. 4. Backward drilling posture in current simulation (left) and in Anton's work (right) 22].

Most previous researches on overhead drilling postures reported a significant difference in efforts demanded by low reach and by high reach 22 33. There are also exceptions. In the study of Maciukiewicz et al. (2016) [32, with the 
same working height, the standing posture with lower arm reach is found to go with more intensive muscle activities in compare with the sitting posture with higher arm height. In current simulation, the lower reach height is estimated to go with a bit more muscular efforts than the higher reach height in the case of far forward reach. The diverse results indicate the complexity of posture analysis. No universe principle could apply to all practical cases. Biomechanical analysis based on detailed posture configuration should be conducted for job design.

Besides, the simulation result also reveals significantly different activation states for various heads of a muscle branch (Table 4), which indicates different roles played by different heads of a muscle. Therefore, it is suggested that future researches avoid vague terms such as biceps, deltoids, but rather precisely down to the head of muscles.

Concerning the study of the muscle fatigue, an activation rate equal to $100 \%$ means the worker cannot keep this posture because his muscle capacities will decrease quickly. Conversely, a low activation rate means the worker can keep longer this posture. During usual tasks, it is often request to be under $20 \%$ of activation rate. So, for this task, only the L-M posture is feasible for drilling operations.

Indeed, if we compare the for the whole body, the estimated muscle activation, the $\mathrm{L}-\mathrm{C}$ and $\mathrm{H}-\mathrm{C}$ postures are mostly the same. However, for the $\mathrm{L}-\mathrm{C}$ posture, maximal activation rate permits to the worker a short activity but not for the $\mathrm{H}-\mathrm{C}$ posture.

This last remark permits us to write a future optimization problem: minimize the whole body muscle activation under constraint to the maximal value of local muscle activation. This problem need to find new realistic human postures and the computation of the inverse dynamic model at any steeps of the computation to determine new muscle activation rates.

\section{Conclusions and future works}

This research introduces a full-chain OpenSim model. It consists of 45 body segments, 424 muscles and 39 degrees of freedom. This model was used to simulate an overhead drilling task. Results suggest that beside of shoulder elevation moment, shoulder rotation moment is also considerable during overhead drilling; and back muscles play an important role. In addition, the total muscle activations vary markedly with different postures for this task. Conclusions are drawn that the full-chain OpenSim model facilities biomechanical analysis in three aspects: i) a comprehensive estimation of multi-degree-of-freedom joints' moments; ii) understanding how the muscles across body cooperate in motions; iii) optimizing working posture in view of total muscle efforts.

Future works will be done to optimize working postures under constraint of maximal muscle activity to minimize the worker fatigue or to adjust the behavior of exosqueleton mechanism attached to the worker. Indeed, a local assistant can remove local muscle constraint but increase the muscle activity, for another part body, and yields to new musculosqueleton disorder. Thus, the full- 
body muscle model with a motion capture system can also predict the dynamic muscle endurance times for complex task where many muscles are activated to produce the motion.

\section{Acknowledgments}

This work was supported by INTERWEAVE Project (Erasmus Mundus Partnership Asia-Europe) under Grants number IW14AC0456 and IW14AC0148, by the National Natural Science Foundation of China under Grant numbers 71471095 and by Chinese State Scholarship Fund.

\section{References}

1. Eurogip: Declaration des maladies professionnelles : problematique et bonnes pratiques dans cinq pays europeens. https://www.eurogip.fr/images/documents/ 3906/Rapport_DeclarationMP_EUROGIP_102F.pdf p44, 2015.

2. Robert, T., Chèze, L., Dumas, R., Verriest, J.P.: Validation of net joint loads calculated by inverse dynamics in case of complex movements: application to balance recovery movements. Journal of biomechanics 40(11) (2007) 2450-2456

3. Delp, S.L., Anderson, F.C., Arnold, A.S., Loan, P., Habib, A., John, C.T., Guendelman, E., Thelen, D.G.: Opensim: open-source software to create and analyze dynamic simulations of movement. IEEE transactions on biomedical engineering 54(11) (2007) 1940-1950

4. Thelen, D.G., Anderson, F.C.: Using computed muscle control to generate forward dynamic simulations of human walking from experimental data. Journal of biomechanics 39(6) (2006) 1107-1115

5. Kim, H.K., Zhang, Y.: Estimation of lumbar spinal loading and trunk muscle forces during asymmetric lifting tasks: application of whole-body musculoskeletal modelling in opensim. Ergonomics 60(4) (2017) 563-576

6. Hamner, S.R., Seth, A., Delp, S.L.: Muscle contributions to propulsion and support during running. Journal of biomechanics 43(14) (2010) 2709-2716

7. Christophy, M., Senan, N.A.F., Lotz, J.C., O?Reilly, O.M.: A musculoskeletal model for the lumbar spine. Biomechanics and modeling in mechanobiology 11(12) (2012) 19-34

8. Holzbaur, K.R., Murray, W.M., Delp, S.L.: A model of the upper extremity for simulating musculoskeletal surgery and analyzing neuromuscular control. Annals of biomedical engineering 33(6) (2005) 829-840

9. Ma, L., Chablat, D., Bennis, F., Zhang, W.: A new simple dynamic muscle fatigue model and its validation. International Journal of Industrial Ergonomics 39(1) (2009) 211-220

10. Ma, L., Chablat, D., Bennis, F., Zhang, W., Guillaume, F.: A new muscle fatigue and recovery model and its ergonomics application in human simulation. Virtual and Physical Prototyping 5(3) (2010) 123-137

11. Ma, L., Zhang, W., Chablat, D., Bennis, F., Guillaume, F.: Multi-objective optimisation method for posture prediction and analysis with consideration of fatigue effect and its application case. Computers \& Industrial Engineering 57(4) (2009) $1235-1246$ 
12. Ma, L., Chablat, D., Bennis, F., Zhang, W., Hu, B., Guillaume, F.: A novel approach for determining fatigue resistances of different muscle groups in static cases. International Journal of Industrial Ergonomics 41(1) (2011) 10-18

13. Sakka, S., Chablat, D., Ma, R., Bennis, F.: Predictive model of the human muscle fatigue: application to repetitive push-pull tasks with light external load. International Journal of Human Factors Modelling and Simulation 5(1) (2015) 81-97

14. Chang, J., Chablat, D., Bennis, F., Ma, L.: Muscle fatigue analysis using opensim. In: International Conference on Digital Human Modeling and Applications in Health, Safety, Ergonomics and Risk Management, Springer (2017) 95-106

15. Raabe, M.E., Chaudhari, A.M.: An investigation of jogging biomechanics using the full-body lumbar spine model: Model development and validation. Journal of biomechanics 49(7) (2016) 1238-1243

16. Saul, K.R., Hu, X., Goehler, C.M., Vidt, M.E., Daly, M., Velisar, A., Murray, W.M.: Benchmarking of dynamic simulation predictions in two software platforms using an upper limb musculoskeletal model. Computer methods in biomechanics and biomedical engineering 18(13) (2015) 1445-1458

17. Chang, J., Chablat, D., Fouad, B., Ma, L., Bennis, F.: Using 3d scan to determine human body segment mass in opensim model. arXiv preprint arXiv:1805.05330, 20th International Conference on Human-computer Interaction (2018)

18. Van Rijn, R.M., Huisstede, B.M., Koes, B.W., Burdorf, A.: Associations between work-related factors and specific disorders of the shoulder?a systematic review of the literature. Scandinavian journal of work, environment \& health (2010) 189-201

19. Bernard, B.P., Putz-Anderson, V.: Musculoskeletal disorders and workplace factors; a critical review of epidemiologic evidence for work-related musculoskeletal disorders of the neck, upper extremity, and low back. (1997)

20. Herberts, P., Kadefors, R., Andersson, G., Petersén, I.: Shoulder pain in industry: an epidemiological study on welders. Acta Orthopaedica Scandinavica 52(3) (1981) 299-306

21. Rempel, D., Star, D., Barr, A., Blanco, M.M., Janowitz, I.: Field evaluation of a modified intervention for overhead drilling. Journal of Occupational and Environmental Hygiene 7(4) (2010) 194-202

22. Anton, D., Shibley, L.D., Fethke, N.B., Hess, J., Cook, T.M., Rosecrance, J.: The effect of overhead drilling position on shoulder moment and electromyography. Ergonomics 44(5) (2001) 489-501

23. Walker, B.F.: The prevalence of low back pain: a systematic review of the literature from 1966 to 1998. Clinical Spine Surgery 13(3) (2000) 205-217

24. Dagenais, S., Caro, J., Haldeman, S.: A systematic review of low back pain cost of illness studies in the united states and internationally. The spine journal 8(1) (2008) 8-20

25. Andersson, G.B.: Epidemiological features of chronic low-back pain. The lancet 354(9178) (1999) 581-585

26. June, K.J., Cho, S.H.: Low back pain and work-related factors among nurses in intensive care units. Journal of Clinical Nursing 20(3-4) (2011) 479-487

27. Tissot, F., Messing, K., Stock, S.: Studying the relationship between low back pain and working postures among those who stand and those who sit most of the working day. Ergonomics 52(11) (2009) 1402-1418

28. Hoogendoorn, W.E., Bongers, P.M., de Vet, H.C., Douwes, M., Koes, B.W., Miedema, M.C., Ariëns, G.A., Bouter, L.M.: Flexion and rotation of the trunk and lifting at work are risk factors for low back pain: results of a prospective cohort study. Spine 25(23) (2000) 3087-3092 
29. Lin, Y.C., Dorn, T.W., Schache, A.G., Pandy, M.G.: Comparison of different methods for estimating muscle forces in human movement. Proceedings of the Institution of Mechanical Engineers, Part H: Journal of Engineering in Medicine 226(2) (2012) 103-112

30. Anderson, F.C., Pandy, M.G.: Static and dynamic optimization solutions for gait are practically equivalent. Journal of biomechanics 34(2) (2001) 153-161

31. Seth, A., Pandy, M.G.: A neuromusculoskeletal tracking method for estimating individual muscle forces in human movement. Journal of biomechanics 40(2) (2007) 356-366

32. Maciukiewicz, J.M., Cudlip, A.C., Chopp-Hurley, J.N., Dickerson, C.R.: Effects of overhead work configuration on muscle activity during a simulated drilling task. Applied ergonomics 53 (2016) 10-16

33. Shin, S.J., Yoo, W.G.: Effects of overhead work involving different heights and distances on neck and shoulder muscle activity. Work 51(2) (2015) 321-326 